\title{
It Could Not Be Seen Because It Could Not Be Believed on June 30, 2013
}

\author{
Fred J. Schoeffler ${ }^{1(\bowtie)}$ and Lance Honda ${ }^{2}$ \\ ${ }^{1}$ Sheff LLC, Pine, USA \\ dougfir777@yahoo.com \\ 2 Prineville, USA \\ Ithonda52@gmail.com
}

\begin{abstract}
Nineteen Prescott Fire Department, Granite Mountain Hot Shot (GMHS) wildland firefighters (WF) perished in Arizona in June 2013 Yarnell Hill Fire, an inexplicable wildland fire disaster. In complex wildland fires, sudden, dynamic changes in human factors and fire conditions can occur, thus mistakes can be unfortunately fatal. Individual and organizational faults regarding the predictable, puzzling, human failures that will result in future WF deaths are addressed. The GMHS were individually, then collectively fixated with abandoning their Safety Zone to reengage, committing themselves at the worst possible time, to relocate to another Safety Zone - a form of collective tunnel vision. Our goal is to provoke meaningful discussion toward improved wildland firefighter safety with practical solutions derived from a long-established wildland firefighter expertise/performance in a fatality-prone profession. Wildfire fatalities are unavoidable, hence these proposals, applied to ongoing training, can significantly contribute to other well-thought-out and validated measures to reduce them.
\end{abstract}

Keywords: Wildland fire $\cdot$ Hot Shot $\cdot$ Human factors $\cdot$ Groupthink

\section{Introduction}

Due to the continuing need for research on the Yarnell Hill (YH) Fire fatalities as well as this paper's focus on the Human Factors and Human Errors, some sections of the current paper may reiterate important inclusions from the authors' previous paper. Additional information and analyses in sufficient detail will be included [1].

The title derives from Human Factors researcher Charles Perrow referring to "data on low-consequence events", showing that organizations may have risk under control, and therefore harbor a perceived "great safety culture", because they have the data to prove it [2]. He says this, “... can encourage a collective sense of invulnerability”, underscoring Janis's seminal work on Groupthink, where a “...warning of an incomprehensible and unimaginable event cannot be seen, because it cannot be believed" [2, 3].

Many of us continue to struggle with convincingly making sense of the June 30, 2013 YH Fire. The "how and why" of 19 of the 20 Granite Mountain Hot Shot (GMHS) fatalities was, is, and always will be, on an unfathomable scale a nagging enigma. Many wildland firefighters (WF) and supervisors saw the GMHS operations as 
exceedingly unsafe. However, most in the wildland firefighting realm never saw this coming, because in the end, no one would have believed it possible that so many men could have died on a wildland fire in one deadly fell swoop.

\subsection{Background}

Wildland firefighting is recognized as "inherently dangerous" [4]. Countless fireline distractions limit discerning conflicting organizational and systems goals, hence avoiding gradual drifts into failure, which stupefies WFs at times. Nineteen GMHS WF, sponsored by the Prescott Fire Department (PFD), perished together on the June 30, 2013 YH Fire in Arizona. This infamous tragedy is accepted by most WFs as the most contentious, heart-rending, inexplicable, and vexing wildland fire disaster. Wildland fire fatalities are inevitabilities based on the interactions of complex environmental, social, cultural, and individual behaviors. Several of the GMHS decisions were critically flawed because of these complexities and their own limitations. Their resolve to vacate their Safety Zone (SZ) was one element in a long chain of bad decisions that led them well beyond safe and tenable boundaries into a fatal fire entrapment [1]. Many career WFs deemed the circumstances of this event as predictable and reasoned, had the GMHS heeded their prescient WF instincts this disaster could have been prevented or at least lessened. However, others who disagree might consider this hindsight bias.

Both individual and organizational faults are considered to better distinguish the predictable, yet puzzling, human failures that will result in future tragic wildland fire deaths [5]. The collective wisdom holds that to lessen such tragedies, sage counsel from numerous authors, researchers, and practitioners, primarily comprised of experienced warriors from the aviation, military, and general firefighting disciplines, must be followed. Their critical and combined guidance underscores the following:

- the significance of continued and repetitive realistic training;

- avoiding feckless safety exhortations, (e.g., "Safety First," and "Stay Safe");

- vigilant assessments by accurate and timely interpretations of warning signs;

- ample and thoughtful mitigation including debating "what ifs";

- refraining from high-risk and little-to no-gain engagements; and

- cautious discernment and professional implementation.

Regarding the YH Fire, the GMHS were first individually and then collectively fixated with abandoning their adequate SZ to unwillingly reengage and commit themselves at the worst possible time. This was allegedly to relocate to the Boulder Springs Ranch (BSR), another SZ [1]. Besides a gap in critical intelligence, this impulsive action likely occurred to alleviate their misperceived notions of non-productivity, evidenced in their text messages. This may have swayed the GMHS leaders' Crew reengagement order to them to assist in the Yarnell evacuations after they had earlier turned down the request [1]. The listed factors below may well have added to faulty decisions:

- cultural influences of municipal fire departments with collateral wildland fire responsibilities to save structures - a form of collective tunnel vision; 
- contentious GMHS leadership and absence of sound leadership;

- weak Crew cohesion using consensus-seeking ("absence of leadership"); and

- marginal to nonexistent situational awareness of changing conditions.

Two examples of GMHS outliers support the authors' above inferences. First, one GMHS, a former Payson Hot Shot (HS), was a quasi-expert regarding wildfire fatality case studies per his former supervisor. Both his girlfriend and his sister were incredulous that this GMHS failed to speak up when the Crew decided to leave "the black". "He knew better," they said [6]. Second, a Rookie GMHS Marine Scout sniper had received intense leadership training in the art of war [7]. His widow believed he would only have left a safe ridge and hiked into danger if directed to do so; he was "... a Marine ... used to following orders" $[1,26]$. The authors contend both men likely recognized the hazardous, impulsive attitudes and "broken conversations" [8] that led to the toxic Groupthink that infected their Crew. Despite these outliers' attempts at dissuasion, the GMHS incredibly left their SZ instead of having cogent group discussions, which may have prevented or lessened the deaths. Section 4.2 covers Groupthink more.

\subsection{Motivation and Goals}

We are told to "prove all things" [9]. The authors assert that the YH Fire Serious Accident Investigation Report (SAIR) was disingenuously labeled as "Factual" by the Serious Accident Investigation Team (SAIT-SAIR) [10]. One notable Human Factors expert, Dr. Ted Putnam, argues that relying on debatable "factual" investigation reports is questionable [11]. This paper's authors strongly contest several of the SAIR assessments of "factual". Inspired to find more hidden truths, the authors critically analyze the dubious SAIT findings and long-established pattern of determining "conclusions" first and then finding "facts" to support them (e.g., the Human Factors Specialist using an idealized image instead a real photo to depict critical fire behavior Fig. 1).

Wildfire fatalities are regrettably unavoidable. "Wildland firefighting is a high-risk occupation, evidenced each year by deaths or injuries in the line of duty;" and we should "... identify factors responsible for past fatalities, [to] mitigate those factors in future fire seasons" [12]. The present authors intend to provoke meaningful discussion among all WFs toward improved Wildland Firefighting safety. Mistakes often occur through the faulty actions of many people; it is mostly the accumulation of many smaller lapses and mistakes that finally result in the bigger error(s) [13]. To meet this goal, this paper:

- identifies WF decision errors specific to the YH Fire;

- applies understanding for training, procedural, and/or systemic changes; and

- analyzes the SAIT-SAIR and further evidence that contest the SAIT findings.

Emphases are placed on the following critical areas:

- Wildland Firefighting Rules

- Environmental Influences

- Human Factors and Human Failure 
- Organizational Cultures and Influences

- Conclusion with Recommendations

Note: The paper's content reflects the views of the authors, both retired HS Crew Superintendents, responsible for the facts and accuracy of the information presented herein. Some content may contain subject matter judged by some to be disturbing.

\section{Wildland Firefighting Rules}

\subsection{General Overview}

All WFs are trained with specific rules, crucial to follow to ensure good direction, leadership, safety, and vigilance. The Standard Firefighting Orders, organized deliberately and sequentially, are to be carried out analytically in all fire situations [14]. The 18 Watch Out Situations, (i.e., guidelines, are faced on all fires, more to warn of impending dangers). The authors and experienced WFs contend knowing and abiding by the wildland firefighting rules works and promotes good, safe decisions and outcomes $[1,13,15,16]$. The authors are firm: there is no known wildfire fatality when all the Standard Firefighting Orders are followed, and the 18 Watch Outs mitigated (10 \& 18).

The most critical of the established Wildland Firefighting Rules are listed in the Incident Response Pocket Guide (NWCG) [17]; 1. Standard Firefighting Orders; 2. Eighteen Watch Out Situations; 3. Downhill Checklist; Lookouts - Communications Escape Routes - Safety Zones (LCES); 5. Common Denominators of Fire Behavior on Tragedy/Near-Miss Fires; and 6. Wildland Urban Interface (WUI) Watch Outs. "If firefighters follow the Standard Firefighting Orders and are alerted to the 18 Watch Out Situations, much of the risk of firefighting can be reduced" [14]. Jointly they are responsible for saving tens of thousands of wildland firefighter lives each-and-every fire season [1]. It is common knowledge and practice in the WF community that these rules work. All firefighters must know, understand, and apply them on every fire, every time despite complaints by some to the contrary $[1,15,16]$. On most wildfire fatalities, questionable actions were initiated, in conflict with several established firefighting decision rules. "Downhill fireline construction is hazardous in steep terrain, fast-burning fuels, or rapidly changing weather" according to human factors researchers [18].

Australian WF created a novel method to address Watch Out \#11 - Unburned fuel between you and the fire [17]. They note: "firefighters engaged in parallel or indirect attack are working in a dead man zone if they do not appreciate the time and space required to find safe refuge." The GMHS were in a 'dead man zone' once they left their refuge [19] and perilously continued downhill in a drainage (chute/chimney) and finally into a deadly box canyon or bowl, all proven potentially deadly, in unburned fuel. 


\subsection{Consequences of Inattention - Fatality Fires and 'Lunch Spot' Nexus Theory}

Additionally, the multi-fatality South Canyon (1994), 30-Mile (2001), and YH (2013) Fires shared a common element when the WFs disengaged to a designated Lunch Spot. In this setting, due to numerous physiological and psychological circumstances, (e.g., fatigue, stress, dehydration, alcohol-related impairment (hangovers), distractions, interruptions, etc.) - time essentially stood still. In each of these above noted tragic wildfires, when the WFs reengaged, they were evidently unmindful of basic wildland firefighting training and fire behavior markers which favored staying put. They were so engrossed with "discussing their options" of staying put or reengaging, they ceased observing more pressing tasks, like the rapidly changing weather. They let go of strategic management for less serious, less vital events, known as strategic omission [20].

Credible research on attention found when someone is otherwise engaged, at times they fail to "see" otherwise noticeable, fully visible, - yet unexpected objects or events, (i.e. 'inattentional blindness' [IB]). A likely severe result is that it can sometimes lead one to miss items that one wanted or, more importantly, needed to experience [21, 22]. If one's attention is set for a certain number of primary-task items and the offered items meet their expectation, the individual may be more likely to exhibit IB for an unforeseen and yet likely critical visual event [21, 22]. IB was driven more by situational and task factors, ... than by individual-differences variables [21]. This may help explain some of why the GMHS and others faced and then reacted to this obscure perilous occurrence.

\section{Environmental Influences}

\subsection{Fuels, Weather, Topography, and Fire Behavior}

Firefighters discuss wildland fires in terms of fuels and what is burning: Fuel type, fuel loading, fuel availability, live and/or dead material including structures. Weather is the most variable, with daytime and the most critical, nighttime temperatures, relative humidity, and winds. Topography is the shape of the earth's surface including its relief and its natural and man-made features, such as steep slopes with drainages, known as chimneys and chutes, slope aspect, and altitude. The last environmental influence, fire behavior, relates to how the fire is currently burning and expected to burn (e.g., smoldering, running, etc.). WFs know the fire always signals its intentions. Briefings and debriefings should always include these topics. The terms are noted and further clarified in the overall Wildland Firefighting Rules in the IRPG [17] and elsewhere [23].

\subsection{Yarnell Hill Fire}

The YH Fire occurred in west-central Arizona west of the town of Yarnell along a north-south mountainous ridge with elevations ranging from 4,500 to 6,000 feet. Within the fire area, the terrain varies from steep ridges to nearly flat valley bottoms 
with numerous rock outcroppings and boulder piles [10]. The terrain was covered with by a dense, volatile grass crop due to prior years' heavy rains with nearly impenetrable, decadent chaparral one to ten feet in height, unburned since 1966, creating an explosive fuel bed with extremely high rates of spread and extreme resistance to control. Arizona had experienced a severe drought for several years and was critically hot and dry in the prior weeks. On other fatal and near-fatal Southwestern fires and prescribed burns, the strongest and most variable winds occur during thunderstorms, and generate extreme downdrafts, micro-bursts, outflows, and gust fronts, that adversely affect fire behavior, thus exacerbating and complicating WF choices, priorities, and safety [1, 10, 24].

The fire began small in difficult terrain in desiccated heavy chaparral fuel and grew exponentially (i.e., when the value of a quantity at any time is its previous value multiplied by a certain number, the same number each time) as the intense outflow downdraft winds increased in both size and complexity, overwhelming the initial and extended attack WFs and the incoming Incident Management Teams (IMT) [5, 24]. Curiously, per the SAIR, "As complexity dramatically increased starting Saturday evening, fire management went through multiple transitions from a Type 4 through a Type 1 incident in fewer than 20 h" [10]. In Sect. 5, the authors stress that the SAIT failed to go further in their investigation and discuss the adverse effects this (mis)management and (in)actions of the entire fire had on the individual and overall WFs' decisions to safely and effectively fight the YH Fire [11]. They may well have examined these indispensable revelatory details, but never published them, which might suggest misfeasance.

\section{Human Factors and Human Failure}

Human factors can be deduced as the cause significant consequences on wildland fires even when only fire behavior evidence is presented [16, 25]. Following the rigid Standard Firefighting Orders, created in the 1950s, and adhering to the cautionary 18 Watch Out Situations, WFs gain proactive measures to reduce severe outcomes [17]. Annually, WFs validate the wildland firefighting rules during required fire refreshers and trainings. It is widely accepted in the wildland firefighting realm that all 19 GMHS could have survived in the YH Fire had they held to these tried-and-true rules $[1,15$, 16, 26]. Challenging the SAIT-SAIR, the present authors contend that the SAIT omitted seriously addressing Human Failure, specifically how, why, and when the GMHS considered their risky actions which had been acceptable for years, thus allowing them to unwisely drift into failure $[11,26]$. Dr. Putnam and others argue that a lack of honest examination of the Human Factors influencing wildfire fatalities is endemic to all SAITs and Reviews dating back to the 1949 Mann Gulch Fire in Montana [1, 11].

\subsection{Individual Blame and Organizational Factors}

Catino (2008) established two distinct tactics for explaining incident origins and dynamics: Individual Blame Logic (IBL) and Organizational Function Logic (OFL). IBL suits societal calls to identify accident cause(s) and transgressor(s) while OFL is an 
organizational and functional tactic, aimed at finding the factors within the system behind event occurrence [5]. In the OFL method, expectations are that similar events will not recur or will infrequently occur once these influences are removed [5]. Both IBL and OFL breaches were present regarding the GMHS, based on a history of bad decisions with good outcomes from 2009 to 2013 [1, 16, 26]. This applies to all other fatal wildfires. Regarding the YH Fire, the authors assert that upper-level GMHS supervisors were liable for their WFs' deaths. Many others in the broader municipal/wildland fire culture may also have indirectly contributed to the GMHS outcomes.

While moving vehicles, the GMHS lookout overheard radio traffic between the Division Supervisor (DIVS-A) and the acting GMHS supervisor, with 17 Crew members, safely atop a ridge. In the radio call, DIVS-A told the GMHS to leave "the [safe] black," and join him at the BSR. The acting GMHS Supt. objected saying such a move would be perilous. This turned into a dispute due to "ambiguity of authority" [18]. The Acting GMHS Supt. told DIVS-A either, "we can't make it" or "we're not going to make it" contrary to the SAIT assertion of a 30-min communication gap [1, $10,26,27]$.

\subsection{Groupthink}

Groupthink symptoms are: (a) illusion of invulnerability, (b) illusion of morality, (c) rationalization, (d) stereotyping, (e) self-censorship, (f) illusion of unanimity, (g) contrarian condemnation, and $(\mathrm{h})$ reliance on cognitive gatekeepers to maintain the status quo [3]. Groups affected by Groupthink often ignore safer alternatives. One of the more famous examples of Groupthink was during the 1996 Mt. Everest Disaster in which six climbers perished [28]. A similar incident was when 2012 Stevens Pass Washington avalanche survivor Michelson noted how difficult it was to break away from the group [8]. On the YH Fire, Groupthink clearly prompted the fatal outcome when the GMHS hiked downhill into a deadly bowl at the worst possible time. During a news conference, the PFD Wildland Battalion Chief (WBC) said he would have followed them blindfolded, that they, “... stuck together" and "... saw and felt the same way", protecting their integrity and their decisions to justify their faulty actions [17, 26]. Similarly, the GMHS Assistant Supt.'s PFD self-evaluation stated: "I think as leaders we need to support each other and back up each other's decisions and actions" [29]. This is true if and only if they are safe, realistic, and achievable, or else they should be discounted and rejected.

\subsection{Willingness to Properly Refuse Risk and Turn Down Protocol}

In the quasi-military wildland fire realm, one is to obey orders with exceptions $[1,26]$. Per the IRPG, a WF is obligated to identify alternative(s) to unsafe assignment(s) and refuse them if needed, a supervisor must tell the WFs that others have refused it. Ideally, this protocol, properly implemented, is vital to effective risk management as it provides timely hazard identification to the chain of command, raises risk awareness for leaders and subordinates, and promotes responsibility [17]. Most times, however, WFs are not told others refused the assignment. Even worse, the WFs that have refused are 
usually transferred to another Division doing irrelevant work, referred to as "Division Siberia", a sector of the fire already extinguished. Most times, those WFs are finally sent back to their home unit with poor evaluations. Most WFs fear utilizing this known essential protocol and would instead likely follow those orders against their better judgement. The Acting GMHS Supt. weakly tried this protocol, however, neither he nor the Crew persuasively employed this option to counter DIVS-A's order to leave their SZ [27].

\subsection{Differences in Leadership Traits - Risk Averse vs. Risk Taking}

Collins and Hansen claim, "In an uncertain and unforgiving environment following the madness of crowds is a good way to get killed" [30]. Accordingly, the GMHS, a large, confident group with a herd mentality, taking unnecessary risks, exemplified this concept. Interestingly, research and empirical data indicate gender plays a role in risk taking. One study regarding computerized wargames, aggression, testosterone, and overconfidence indicates most females were risk averse, compared to their more risk-prone male counterparts [31]. A realistic example was when avalanche survivor Michelson felt she could have convinced the other skiers to discontinue if only she had stayed with them [8]. Another empirical example is of a female supervisor who, on the 1994 South Canyon Fire, refused an eventual deadly downhill fireline assignment [18]. Researchers purport males are mostly identified as "serial" thinkers, who construct tiered plans of action and process data one step at a time - aspects of catalogued thought. Conversely, females, identified as "parallel" thinkers, can achieve many similar simple tasks at once, therefore recognized as more capable of dealing with complex issues. Thus, possibly intervening in option discussions, hence able to avert similar disasters [22, 31].

\subsection{Plan Continuation and Confirmation Biases}

Plan continuation bias is the notion of pressing on when things were beginning to go bad very near one's destination. This may impede a crew's ability to recognize they need to change their course of action, a very powerful, unconscious cognitive preference. This bias may be especially strong during a transition phase and might influence a WF's ability to notice subtle cues indicating that the original conditions have changed [32]. WFs are very mission oriented. However, as stress increases, particularly during deteriorating weather and increasing fire behavior, the ability to dispassionately evaluate the situation, accept the reality that conditions are unfavorable and decide to abort the mission drops off sharply the closer one is to their destination. The closer one is to this end-point, the more one reveals one's tenacious resolve to proceed.

\subsection{Decision-Making Under Stress}

Under intense pressures and stress, one falls back on familiar habits. Following are three examples. On the 1994 South Canyon Fire, the Incident Commander (IC) sharpened his chainsaw rather than managing his resources in critical conditions. On the 2011 30-Mile Fire, the Crew Boss/IC, during increased fire behavior, mopped 
up instead of managing his Crew and resources [1,33]. On the YH Fire, the GMHS, cut off by the main fire as they attempted to relocate to the BSR, mistakenly referred to themselves on the Air-to-Ground (A/G) channel as "Granite Mountain 7" [10]. There is no Granite Mountain 7, only a former PFD "Crew 7" Fuels Crew. Stress can impair understanding situational complexity and the ability to logically strategize. Grossman and others offer workable solutions: frequent, quasi-military drills extensively for "stress acclimatization", "stress inoculation", to "push the envelope ... repeated over time" [22, 34].

\section{Organizational Culture and Influences}

\subsection{Municipal FD and PFD Attitude and Influence}

The PFD WBC truly considered the GMHS his sons. He defended the GMHS fatal (in) actions as well as the PFD (in)actions in a July 2013 News Conference at the YH Fire Fatality Site. The WBC held structure protection as a higher priority over WF safety, “... no WF is satisfied stuck sitting in a SZ while structures were being threatened" [1, 26]. These are strong indicators of the conflicting values of many Municipal FDs that also fight wildfires. Confusion, frustration, and doubt, known hazardous barriers, may support potential human failures on wildfires when structures are threatened. GMHS McDonough's actions are a prime example of the above cultural influences on municipal/wildland FDs and WFs. During the investigation he was asked if the GMHS knew of and followed the " 10 \& 18". Probably coached, he stated, "We had issues with \#10, (Fight fire aggressively having provided for safety first), it's old, that's hillbilly ... we're a lot smarter than that". Third year WFs would not likely conclude that on their own $[17,26]$ So it is quite telling that this influential, spoken PFD municipal/wildland fire "policy" attitude, even though unwritten, was at least acceptably regarded and obeyed by a third year WF. And it would also be a permissible inference that other GMHS accepted and regarded this PFD "policy" attitude as well [22].

\subsection{Critical Examination of the SAIT and SAIR}

High Reliability Organizations (HRO) (e.g., aircraft carrier, nuclear power plant, and wildland fire operations, are preoccupied with all failures, even small ones. Trivial errors discovered early are warning signals of deepening trouble, insight into the health of the entire system. Problems found early allow more possible ways to deal with them. However, non-HRO members tend to overlook their failures, suggesting incompetence, and focus instead on successes, conveying competence. [35]. For many WFs, this is theoretical guidance, and not realistic, evidenced by the repeated fatalities for the same fatal causes - poor decisions based on several physiological and psychological factors ignored by almost all SAITs discussed in this paper.

In fact, in 1996 on the Hochderffer Hills Fire Shelter Deployment Investigation, the Human Factors Specialist briefing began with: "The first thing we are going to do is establish a conclusion, then find the facts to fit that conclusion." The primary author was the Operations Specialist [26] and directly participated in this briefing. The authors 
contend the YH Fire SAIT followed the same format of establishing the conclusion first by finding, “... no indication of negligence, reckless actions, or violations of policy or protocol" $[1,10]$. Affirmatively, their conclusion implies the GMHS safely accomplished their tasks, yet a disaster beyond belief occurred. It is impossible that everything was performed correctly on a wildfire if 19 men burned to death. The SAIT's approving conclusion reflects Orwellian Doublespeak and worse, Doublethink, simultaneously holding two contradictory beliefs as true [29]. The authors and others argue, this ongoing truth manipulation occurs with all wildfire SAIT, SAIR, and other versions of Incident Analyses, Guidance, and Review where WFs are killed by fire $[1,11,26]$.

Consider now the comparison of the idealized image, a fabricated 'conclusion first', with an actual photo that reveals the truth of what factually occurred. Figure 1 below (left image) is the SAIT/SAIR Fig. 18 [10], sham idealized image of the alleged fire split, trapping the GMHS. The SAIR correctly noted the thunderstorm out-flow boundary moved from north to south between 1618 to $1630 \mathrm{MST}$. The fire was "immediate and impressive" and when the fire spread direction changed 180 degrees, intensified when flame lengths doubled while the rates of spread tripled and "problematic fire behavior became extreme" [10]. The authors believe the idealized image falsely alleges, "... the fire entered the middle bowl and moved southwest." The Fig. 1 right photo clearly reveals otherwise. Figure 1's left idealized image shows fire running across the middle bowl, headed for the box canyon behind the "Granite Knoll". In contrast, in the right photo, left of the smoke column and the advancing fire, the backdrop 'hazy' mountain ridge is likely the southern route the GMHS took to reach the saddle and then drop into the box canyon, $540 \mathrm{~s}$ (less than $9 \mathrm{~min}$ ) before the Acting GMHS Supt. first called "Mayday" on A/G. The right photo reveals no evidence that a line of fire was simultaneously 'crawling up' the back ridge toward the saddle,

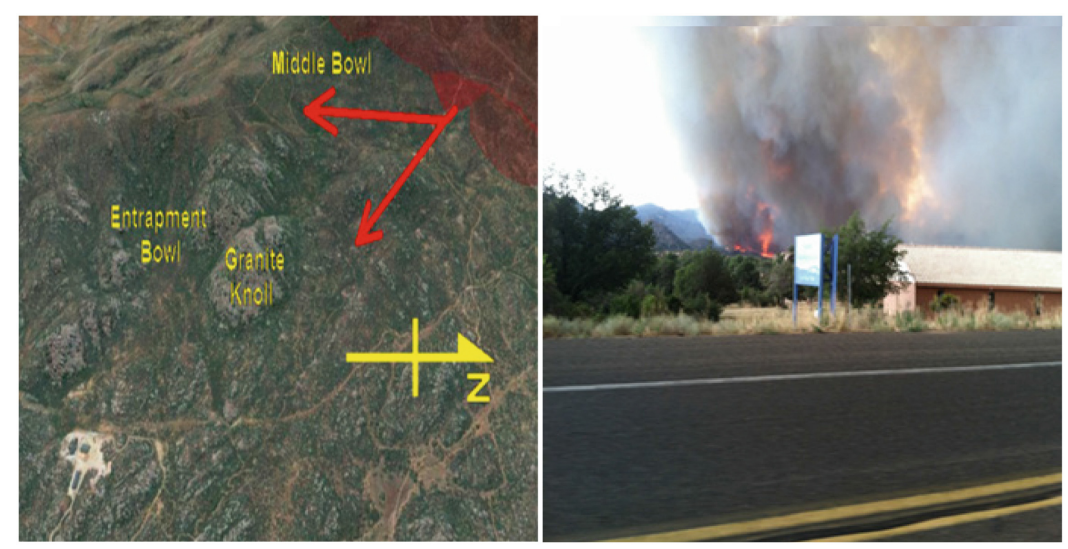

Fig. 1. Left - SAIR Fig. 18 idealized image created by the SAIT [10]. Right - 'iPhone 4' photo taken by AZ Forestry Brian Lauber at 1629 on 6/30/13 near the Yarnell Ranch House restaurant looking west [36]. This photo was provided to Brad Mayhew, SAIT Human Factors Investigator, however, it was not included in the SAIR. 
refuting the SAIT 'theory' that the GMHS saw fire "ahead of them" and "behind them" at 4:39 PM, when the first "Mayday" went out [10, 36]. The authors claim that the SAIT intentionally deceives with this half-truth, “... the middle bowl, ... funneled the fire at a rapid rate of spread toward the top of the ridge" [10]. The photo noticeably contradicts that claim.

\section{Conclusion and Recommendations}

Dorner states: "Our minds have extreme trouble tackling problems that cannot be visualized [24]. Specific concepts for improved wildfire safety and investigations were presented, derived from a long-established and recognized fatality-prone profession. These proposals applied to well-established and dependable ongoing leadership, human factors, and wildfire training, can significantly contribute to validated safety measures to reduce wildfire fatalities. Groupthink and mitigation measures are examined. Burnette et al. found that common goal pursuit under authoritative leadership in a provocative situation can lead groups to take excessive risks. Further research is vital to understand group decision-making, so that WF leaders at all levels, but especially those on the firelines, may recognize and then terminate Groupthink's toxic effects to avert disasters.

The GMHS leaders most directly responsible for Crew actions faced a series of decisions during their ill-fated time on the YH Fire, and their poor decisions brought them to disaster's threshold. Useem et al. state: "The human consequences of suboptimal decisions by fire leaders are compellingly clear, and, conversely, optimal leadership decisions are ... vital for successfully suppressing a fire" [18] and managing WFs. The authors present nine recommendations below to guide WFs to make good, sound, and safe decisions to realize those optimal goals and make history by lessening fatalities.

1. Ensure leadership goals and duties are the safety and welfare of those you supervise;

2. Train beyond sufficiency. Train to warrior status with "stress inoculation";

3. Maintain operational Leadership and Group Dynamics training for all WF's;

4. Reduce Groupthink risks with Leader's Intent, then allow the Group to decide;

5. Hire able, qualified females in supervisory positions to ease Groupthink potential;

6. Censure freelancing - unilaterally committing to tasks minus supervisory consent;

7. Give positive advice and focus, (i.e. tell people what to do instead of what not to do);

8. Demand WF's learn and apply the wildland firefighting rules on every wildfire; and

9. Require absolute honest answers in SAITs and Reviews with fact-led conclusions.

Prior to applying these wildland firefighting insights into practice, cogent, open, ongoing, respectful, and organized empirical research is needed. In spite of the intractable controversial and emotional attitudes and behaviors of many WFs and GMHS family, friends, and loved ones that oppose revealing the YH Fire truths, the authors hope this paper encourages further YH Fire disaster assessments and revelations to better ascertain the many veiled facts and analyses, thus permitting valuable lessons learned. 
Acknowledgements. The authors wish to express their gratitude to: Dr. Martha Underwood and Dr. Katie Orozco for their proficient knowledge and skills in managing this paper; Dr. Ted Putnam for his Human Factors insight; Joy 'Desert Walker' Collura for her rare and priceless YH Fire insight; WantsToKnowTheTruth; Woosdman; Gary Olson; sources mandated silence due to Department and/or Agency 'direction', and sources that choose silence. To those who lost family, friends, and loved ones, the authors and most WFF think about those young men daily. They inspired us to write.

\section{References}

1. Schoeffler, F.J., Honda, L.: Epic human failure on June 30, 2013. In: Boring, R.L. (ed.) Advances in Human Error, Reliability, Resilience, and Performance, 589 p. Springer International Publishing AG 2018 (2016)

2. Perrow, C.: Normal Accidents: Living with High-Risk Technologies, 451 p. Princeton University Press, Princeton (1999)

3. Janis, I.: Victims of Groupthink: A Psychological Study of Foreign Policy Decisions and Disasters, 276 p. Houghton Mifflin, Boston (1972)

4. U.S. Forest Service: Fire Suppression: Foundational Doctrine, 21 p. (2005)

5. Catino, M.: A review of literature: individual blame vs. organizational function logics in accident analysis. J. Conting. Crisis Manage. 16, 53-62 (2008)

6. Dickman, K.: On the Burning Edge: A Fateful Fire and the Men Who Fought It, 277 p. Ballantine Books, New York (2015)

7. Sauter, J.A., Cpl: Scout snipers engage, learn art of leadership U.S. Marines official website, 15 February 2013. Online article (2013)

8. Michelson, M.: Tunnel Vision. Outside Magazine, 10 p. (2012)

9. Barker, K., (ed.): The Zondervan King James Study Bible, 1 Thessalonians 5:21, 2884 p. Grand Rapids, Zondervan (2002)

10. Yarnell Hill Fire Serious Accident Investigation Team (SAIT): Yarnell Hill Fire Serious Accident Investigation Report (SAIR), 122 p. (2013)

11. Putnam, T.: Accidents, accident guides, stories, and the truth. In: Proceedings 11th International Wildland Fire Safety Summit, 4-8 April 2001, 30 p. International Association of Wildland Fire, Missoula, MT, USA (2011)

12. Mangan, R.: Wildland firefighter fatalities in the United States: 1990-2006. NWCG, PMS 841. Boise, ID. National Wildfire Coordinating Group, Safety and Health Working Team, National Interagency Fire Center, 28 p. (2007)

13. Reason J.T.: Managing the Risks of Organizational Accidents, 252 p. Ashgate Publishing, Aldershot, UK (1997)

14. U.S.D.A. Forest Service, Fire and Aviation Management: Risk Management (2017)

15. Coolidge, H.: Rules and Risk in Wildland Firefighting. Hypocrite Reader, vol. 52 (2015)

16. Holmstrom, M.: Common Denominators on Tragedy Fires - Updated for a New (Human) Fire Environment, pp. 26-34. Wildfire Magazine, International Association of Wildland Fire (2016)

17. National Wildfire Coordinating Group (NWCG), Incident Response Pocket Guide (IRPG). PMS 461, NFES 001077 (2014)

18. Useem, M., Cook, J., Sutton, L.: Developing leaders for decision making under stress: wildland firefighters in the South Canyon fire and its aftermath. Acad. Manage. Learn. Educ. 4, 461-485 (2005) 
19. Cheney, P., Gould, J., McCaw, L.: The Dead-Man Zone - a neglected area of firefighter safety. Aust. Forest. 64, 45-50 (2013)

20. Loukopoulos, L.D, Dismukes, R.K, Barshi, I.: The Myth of Multitasking, 188 p. Ashgate, USA (2009)

21. Kreitz, C., Furley, P., Memmert, D., Simons, D.J.: Inattentional blindness and individual differences in cognitive abilities. PLoS ONE 10, e0134675 (2015)

22. Snook, S.A.: Friendly Fire: The Accidental Shootdown of U.S. Black Hawks Over Northern Iraq, 257 p. Princeton Univ. Press, Princeton (2002)

23. Schnepf, C.: Appendix B. The Effects of Topography, Weather, and Fuel on Fire Behavior, 3 p. Oregon State University Library (2017)

24. Dorner, D.: The Logic of Failure: Why Things Go Wrong and What We Can Do to Make Them Right, 122 p. Metropolitan Books, Holt and Co., Inc., New York (1989)

25. Wilson, C.: Fatal and near-fatal forest fires: the common denominators. Intl. Fire Chief $\mathbf{4 3}$, 9-10, 12-15 (1977)

26. Schoeffler, F.J.: Human factors influenced the 30 June 2013 YH Fire Fatalities. Central AZ Wildfire Response Team, RT-130 Refresher. PDF. Academia.edu, 48 p. (2016)

27. Anglen, R., Wagner, D., Sanchez, Y.W.: Yarnell fire: new account of hotshot deaths. Online article, Arizona Republic (2015)

28. Burnette, J.L., Pollack, J.M., Forsyth, D.R.: Analysis of the May 1996 Mount Everest Disaster. J. Leadersh. Stud. 4, 29-40 (2011)

29. Prescott Fire Department. Jesse Steed employee records, Personal Evaluation (2011)

30. Collins, J., Hansen, M.T.: Great by Choice. Uncertainty, Chaos, and Luck: Why Some Thrive Despite Them All, 304 p. Harper Collins Publishers, New York (2011)

31. Johnson, D.D.P., McDermott, R., Barrett, E.S., Cowden, J., McIntyre, M.H., Rosen, S.P.: Overconfidence in wargames: experimental evidence on expectations, aggression, gender and testosterone. Proc. Roy. Soc. B Biol. Sci. 273, 2513-2520 (2006)

32. Dismuke, K., Loukopoulos, L.: The Limits of Expertise: The Misunderstood Role of Pilot Error in Airline Accidents. NASA Human Systems and Flight Cognition (2004)

33. Wildland Fire Lessons Learned Center (LLC) - Incident Reviews, Online (2018)

34. Grossman Dave Lt. Col.: On Combat, 3rd edn., 403 p. Warrior Science Publications (2008)

35. Weick, K.E.: Organizing for transient reliability: organizing for transient reliability: the production of dynamic non-events. J. Conting. Crisis Manage. 19, 21-27 (2011)

36. ASFD/Miscellaneous Photos (IMG_1334.JPG, IMG_1335.JPG and IMG_1336.JPG)

37. https://www.dropbox.com/sh/02ue6bnjp6nazkm/AACyU0pkm-9gu9cJqQNjhnb7a/Photos\% 20and\%20Video/Brian\%20Lauber\%20Photos?dl=0 